\title{
Taut Distance-Regular Graphs of Odd Diameter
}

\author{
MARK S. MACLEAN \\ University of North Carolina, Asheville, NC 28804, USA
}

Received November 28, 2000; Revised June 7, 2002

Abstract. Let $\Gamma$ denote a bipartite distance-regular graph with diameter $D \geq 4$, valency $k \geq 3$, and distinct eigenvalues $\theta_{0}>\theta_{1}>\cdots>\theta_{D}$. Let $M$ denote the Bose-Mesner algebra of $\Gamma$. For $0 \leq i \leq D$, let $E_{i}$ denote the primitive idempotent of $M$ associated with $\theta_{i}$. We refer to $E_{0}$ and $E_{D}$ as the trivial idempotents of $M$. Let $E, F$ denote primitive idempotents of $M$. We say the pair $E, F$ is taut whenever (i) $E, F$ are nontrivial, and (ii) the entry-wise product $E \circ F$ is a linear combination of two distinct primitive idempotents of $M$. We show the pair $E, F$ is taut if and only if there exist real scalars $\alpha, \beta$ such that

$$
\sigma_{i+1} \rho_{i+1}-\sigma_{i-1} \rho_{i-1}=\alpha \sigma_{i}\left(\rho_{i+1}-\rho_{i-1}\right)+\beta \rho_{i}\left(\sigma_{i+1}-\sigma_{i-1}\right) \quad(1 \leq i \leq D-1),
$$

where $\sigma_{0}, \sigma_{1}, \ldots, \sigma_{D}$ and $\rho_{0}, \rho_{1}, \ldots, \rho_{D}$ denote the cosine sequences of $E, F$, respectively. We define $\Gamma$ to be taut whenever $\Gamma$ has at least one taut pair of primitive idempotents but $\Gamma$ is not 2-homogeneous in the sense of Nomura and Curtin. Assume $\Gamma$ is taut and $D$ is odd, and assume the pair $E, F$ is taut. We show

$$
\begin{aligned}
\frac{\sigma_{i+1}-\alpha \sigma_{i}}{\sigma \sigma_{i}-\sigma_{i-1}} & =\frac{\beta \rho_{i}-\rho_{i-1}}{\rho \rho_{i}-\rho_{i-1}} \\
\frac{\rho_{i+1}-\beta \rho_{i}}{\rho \rho_{i}-\rho_{i-1}} & =\frac{\alpha \sigma_{i}-\sigma_{i-1}}{\sigma \sigma_{i}-\sigma_{i-1}}
\end{aligned}
$$

for $1 \leq i \leq D-1$, where $\sigma=\sigma_{1}, \rho=\rho_{1}$. Using these equations, we recursively obtain $\sigma_{0}, \sigma_{1}, \ldots, \sigma_{D}$ and $\rho_{0}, \rho_{1}, \ldots, \rho_{D}$ in terms of the four real scalars $\sigma, \rho, \alpha, \beta$. From this we obtain all intersection numbers of $\Gamma$ in terms of $\sigma, \rho, \alpha, \beta$. We showed in an earlier paper that the pair $E_{1}, E_{d}$ is taut, where $d=(D-1) / 2$. Applying our results to this pair, we obtain the intersection numbers of $\Gamma$ in terms of $k, \mu, \theta_{1}, \theta_{d}$, where $\mu$ denotes the intersection number $c_{2}$. We show that if $\Gamma$ is taut and $D$ is odd, then $\Gamma$ is an antipodal 2-cover.

Keywords: distance-regular graph, association scheme, bipartite graph, tight graph, taut graph

\section{Introduction}

Let $\Gamma$ denote a distance-regular graph with diameter $D \geq 4$ and valency $k \geq 3$. Let $M$ denote the Bose-Mesner algebra of $\Gamma$. It is well-known that $M$ has a basis consisting of primitive idempotents; we refer to these as the primitive idempotents of $\Gamma . M$ is closed under the entry-wise product, so given primitive idempotents $E, F$ of $\Gamma$, the entry-wise product $E \circ F$ is a linear combination of the primitive idempotents of $\Gamma$. The coefficients in this linear combination are the Krein parameters of $\Gamma$. We are interested in the case where many of these coefficients are zero, so $E \circ F$ is a linear combination of a small number of primitive idempotents. For example, suppose $\Gamma$ is $Q$-polynomial relative to $E$. Then $E \circ F$ is a linear combination of at most three primitive idempotents of $\Gamma$. For a related example, suppose 
the above $Q$-polynomial structure is dual bipartite in the sense of Dickie and Terwilliger [4]. Then $E \circ F$ is a linear combination of at most two primitive idempotents of $\Gamma$.

Motivated by these examples, we study those pairs $E, F$ where $E \circ F$ is a linear combination of at most two primitive idempotents of $\Gamma$. We summarize what is known so far. To do this, we use the following notation. Let $k=\theta_{0}>\theta_{1}>\cdots>\theta_{D}$ denote the distinct eigenvalues of $\Gamma$, and for $0 \leq i \leq D$, let $E_{i}$ denote the primitive idempotent of $\Gamma$ associated with $\theta_{i}$. A primitive idempotent of $\Gamma$ will be called trivial whenever it has rank 1 . If $\Gamma$ is not bipartite, then $E_{0}$ is the unique trivial primitive idempotent of $\Gamma$. If $\Gamma$ is bipartite, then $E_{0}, E_{D}$ are the only trivial primitive idempotents of $\Gamma$. Let $E, F$ denote primitive idempotents of $\Gamma$. By [1, Props. II.3.7, II.3.8], $E \circ F$ is not zero, so $E \circ F$ is a linear combination of at least one primitive idempotent of $\Gamma$. In some cases, $E \circ F$ is a scalar multiple of a primitive idempotent of $\Gamma$. For example, suppose at least one of $E, F$ is trivial. Then $E \circ F$ is a scalar multiple of a primitive idempotent of $\Gamma$. We say the pair $E, F$ is tight whenever (i) $E, F$ are nontrivial, and (ii) $E \circ F$ is a scalar multiple of a primitive idempotent of $\Gamma$. In [11], Jurišić, Koolen and Terwilliger introduce the notion of a tight distance-regular graph. Pascasio proves in [18, Theorem 1.3] that $\Gamma$ is tight if and only if $\Gamma$ has at least one tight pair of primitive idempotents. In this paper, we define $\Gamma$ to be tight whenever $\Gamma$ has at least one tight pair of primitive idempotents.

Suppose $\Gamma$ is tight. Then by a result of Pascasio [18], $\Gamma$ is not bipartite. Moreover, the pair $E, F$ is tight if and only if $E, F$ is a permutation of $E_{1}, E_{D}$. See $[10,11]$ for a detailed discussion of the tight graphs. For related papers, see [6-9, 15-19].

Now we consider the case where $E \circ F$ is a linear combination of two distinct primitive idempotents of $\Gamma$. To keep things simple, we restrict our attention to the case where $\Gamma$ is bipartite. For the rest of this introduction, assume $\Gamma$ is bipartite. We define the pair $E, F$ to be taut whenever (i) $E, F$ are nontrivial, and (ii) $E \circ F$ is a linear combination of two distinct primitive idempotents of $\Gamma$. There are a few ways a pair of primitive idempotents can be taut.

Suppose $\Gamma$ is 2-homogeneous in the sense of Nomura [13] and Curtin [3]. In this case, we showed in [12] that the pair $E, F$ is taut if and only if $E, F$ are nontrivial with at least one equal to $E_{1}$ or $E_{D-1}$.

We define $\Gamma$ to be taut whenever $\Gamma$ is not 2-homogeneous and there exists at least one taut pair of primitive idempotents of $\Gamma$. Suppose $\Gamma$ is taut and $D$ is odd. In [12], we showed the pair $E, F$ is taut if and only if the set $\{E, F\}$ is one of $\left\{E_{1}, E_{d}\right\},\left\{E_{1}, E_{d+1}\right\},\left\{E_{D-1}, E_{d}\right\},\left\{E_{D-1}\right.$, $\left.E_{d+1}\right\}$, where $d=(D-1) / 2$. Now suppose $\Gamma$ is taut and $D$ is even. In [12], we showed the pair $E, F$ is taut if and only if the set $\{E, F\}$ is one of $\left\{E_{1}, E_{d}\right\},\left\{E_{D-1}, E_{d}\right\}$, where $d=D / 2$.

We now summarize our results in the present paper. Let $\Gamma$ denote a bipartite distanceregular graph with diameter $D \geq 4$, valency $k \geq 3$, and eigenvalues $\theta_{0}>\theta_{1}>\cdots>$ $\theta_{D}$. Let $E, F$ denote nontrivial primitive idempotents of $\Gamma$, and let $\sigma_{0}, \sigma_{1}, \ldots, \sigma_{D}$ and $\rho_{0}, \rho_{1}, \ldots, \rho_{D}$ denote the corresponding cosine sequences. We show the following are equivalent: (i) the pair $E, F$ is taut, and (ii) there exist complex scalars $\alpha, \beta$ such that

$$
\sigma_{i+1} \rho_{i+1}-\sigma_{i-1} \rho_{i-1}=\alpha \sigma_{i}\left(\rho_{i+1}-\rho_{i-1}\right)+\beta \rho_{i}\left(\sigma_{i+1}-\sigma_{i-1}\right)
$$

for $1 \leq i \leq D-1$. Moreover, if (i), (ii) hold, then $\alpha, \beta$ are real. 
We now suppose $\Gamma$ is taut and $D$ is odd. Further suppose the pair $E, F$ is taut. Using (1), we show

$$
\begin{aligned}
\frac{\sigma_{i+1}-\alpha \sigma_{i}}{\sigma \sigma_{i}-\sigma_{i-1}} & =\frac{\beta \rho_{i}-\rho_{i-1}}{\rho \rho_{i}-\rho_{i-1}}, \\
\frac{\rho_{i+1}-\beta \rho_{i}}{\rho \rho_{i}-\rho_{i-1}} & =\frac{\alpha \sigma_{i}-\sigma_{i-1}}{\sigma \sigma_{i}-\sigma_{i-1}}
\end{aligned}
$$

for $1 \leq i \leq D-1$, where $\alpha, \beta$ are from (1), and where $\sigma=\sigma_{1}, \rho=\rho_{1}$. Using (2), (3), we recursively obtain $\sigma_{0}, \sigma_{1}, \ldots, \sigma_{D}$ and $\rho_{0}, \rho_{1}, \ldots, \rho_{D}$ in terms of the four real scalars $\sigma, \rho, \alpha, \beta$. From this we obtain all intersection numbers of $\Gamma$ in terms of $\sigma, \rho, \alpha, \beta$. Applying this result to the taut pair $E_{1}, E_{d}$, we obtain the intersection numbers of $\Gamma$ in terms of the four parameters $k, \mu, \theta_{1}, \theta_{d}$, where $\mu$ denotes the intersection number $c_{2}$.

Finally, we show that if $\Gamma$ is taut and $D$ is odd, then $\Gamma$ is an antipodal 2-cover.

\section{Preliminaries}

In this section, we set our notation and review some basic definitions and results. For more information, the reader may consult the books of Bannai and Ito [1], Brouwer et al. [2], and Godsil [5].

Throughout this paper, let $\Gamma$ denote a distance-regular graph with vertex set $X$ and diameter $D$. As usual, we let $p_{i j}^{h}(0 \leq h, i, j \leq D)$ denote the intersection numbers of $\Gamma$. It is conventional to abbreviate $c_{i}:=p_{1 i-1}^{i}(1 \leq i \leq D), a_{i}:=p_{1 i}^{i}(0 \leq i \leq D), b_{i}:=p_{1 i+1}^{i}$ $(0 \leq i \leq D-1), k_{i}:=p_{i i}^{0}(0 \leq i \leq D)$, and to define $c_{0}:=0, b_{D}:=0$. For convenience, we write $\mu:=c_{2}$. By [2, p. 127] we have

$$
k_{i}=\frac{b_{0} b_{1} b_{2} \cdots b_{i-1}}{c_{1} c_{2} \cdots c_{i}} \quad(0 \leq i \leq D)
$$

We let $A_{0}, A_{1}, \ldots, A_{D} \in \operatorname{Mat}_{X}(\mathbb{C})$ denote the distance matrices of $\Gamma$. The matrix $A_{1}$ is the adjacency matrix of $\Gamma$; we frequently abbreviate $A:=A_{1}$. We let $M$ denote the BoseMesner algebra of $\Gamma$, which is the subalgebra of $\operatorname{Mat}_{X}(\mathbb{C})$ generated by $A$. It is well-known that the distance matrices form a basis for $M$. We let $E_{0}, E_{1}, \ldots, E_{D}$ denote the primitive idempotents of $M$ (we frequently refer to these matrices as the primitive idempotents of $\Gamma$ ), where $E_{0}$ is a scalar multiple of the all 1 's matrix. The primitive idempotents $E_{0}, E_{1}, \ldots, E_{D}$ also form a basis for $M$. For $0 \leq i \leq D$, we let $m_{i}:=\operatorname{rank}\left(E_{i}\right)$ denote the multiplicity of $E_{i}$.

There exist distinct real numbers $\theta_{0}, \theta_{1}, \ldots, \theta_{D}$ such that $A=\sum_{i=0}^{D} \theta_{i} E_{i}$. We say that $\theta_{i}$ is the eigenvalue of $\Gamma$ associated with $E_{i}$. One can show $\theta_{0}=k$ and $-k \leq \theta_{i} \leq k$ for $0 \leq i \leq D$ [1, Thm. III.1.3].

Let $E$ denote a primitive idempotent of $\Gamma$, and let $m$ denote the multiplicity of $E$. By [1, Section II.3], there exist real scalars $\sigma_{0}, \sigma_{1}, \ldots, \sigma_{D}$ such that $\sigma_{0}=1$ and

$$
E=|X|^{-1} m \sum_{i=0}^{D} \sigma_{i} A_{i} .
$$


The sequence $\sigma_{0}, \sigma_{1}, \ldots, \sigma_{D}$ is called the cosine sequence of $\Gamma$ associated with $E$. We abbreviate $\sigma:=\sigma_{1}$ and refer to $\sigma$ as the first cosine of $E$. Let $\theta_{0}>\theta_{1}>\cdots>\theta_{D}$ denote the eigenvalues of $\Gamma$, and let $\sigma_{0}, \sigma_{1}, \ldots, \sigma_{D}$ denote the cosine sequence associated with $\theta_{1}$. Then by [5, Section 13, Lemma 2.1],

$$
\sigma_{0}>\sigma_{1}>\cdots>\sigma_{D}
$$

Since each entry in the distance matrices is either 0 or 1 , we have

$$
A_{i} \circ A_{j}=\delta_{i j} A_{i} \quad(0 \leq i, j \leq D)
$$

where $\circ$ denotes the entry-wise matrix product. It follows that $M$ is closed under $\circ$. In particular, there exist scalars $q_{i j}^{h} \in \mathbb{C}$ such that

$$
E_{i} \circ E_{j}=|X|^{-1} \sum_{h=0}^{D} q_{i j}^{h} E_{h} \quad(0 \leq i, j \leq D) .
$$

The scalars $q_{i j}^{h}$ are known as the Krein parameters of $\Gamma$. The Krein parameters are real and nonnegative [2, Theorem 2.3.2]. From [2, Lemma 2.3.1], we have

$$
q_{i j}^{0}=\delta_{i j} m_{i} \quad(0 \leq i, j \leq D)
$$

In this paper we will be considering pairs of primitive idempotents $E_{i}, E_{j}$ such that $E_{i} \circ E_{j}$ is a linear combination of one or two primitive idempotents. Observe the existence of such a pair $E_{i}, E_{j}$ implies that a number of the Krein parameters are zero.

For the rest of this section, we recall some facts about bipartite distance-regular graphs. Let $\Gamma$ denote a bipartite distance-regular graph with diameter $D$, valency $k$, and eigenvalues $\theta_{0}>\theta_{1}>\cdots>\theta_{D}$. It is well-known that $a_{i}=0(0 \leq i \leq D)$, and so $c_{i}+b_{i}=k$ $(0 \leq i \leq D)$ [2, Prop. 4.2.2]. Furthermore, one can show

$$
\theta_{D-i}=-\theta_{i} \quad(0 \leq i \leq D) .
$$

Lemma 2.1 ([2, p. 128]) Let $\Gamma$ denote a bipartite distance-regular graph with diameter $D \geq 3$. For any complex scalars $\sigma_{0}, \sigma_{1}, \ldots, \sigma_{D}$, the following are equivalent.

(i) $\sigma_{0}, \sigma_{1}, \ldots, \sigma_{D}$ is a cosine sequence of $\Gamma$.

(ii) $\sigma_{0}=1, \sigma_{D-1}=\sigma \sigma_{D}$, and

$$
c_{i}\left(\sigma_{i-1}-\sigma_{i+1}\right)=k\left(\sigma \sigma_{i}-\sigma_{i+1}\right) \quad(1 \leq i \leq D-1) .
$$

(iii) $\sigma_{0}=1, \sigma_{D-1}=\sigma \sigma_{D}$, and

$$
b_{i}\left(\sigma_{i+1}-\sigma_{i-1}\right)=k\left(\sigma \sigma_{i}-\sigma_{i-1}\right) \quad(1 \leq i \leq D-1) .
$$

Furthermore, suppose (i)-(iii) hold. Then $\theta=k \sigma$ is the eigenvalue of $\Gamma$ associated with the cosine sequence $\sigma_{0}, \sigma_{1}, \ldots, \sigma_{D}$. 
Corollary 2.2 Let $\Gamma$ denote a bipartite distance-regular graph with diameter $D \geq 3$, and let $\sigma_{0}, \sigma_{1}, \ldots, \sigma_{D}$ denote a cosine sequence of $\Gamma$. Then for any integer $i(1 \leq i \leq D-1)$, the following are equivalent.

(i) $\sigma_{i+1}=\sigma_{i-1}$.

(ii) $\sigma \sigma_{i}=\sigma_{i-1}$.

(iii) $\sigma \sigma_{i}=\sigma_{i+1}$.

Corollary 2.3 Let $\Gamma$ denote a bipartite distance-regular graph with diameter $D \geq 3$. Let $\sigma_{0}, \sigma_{1}, \ldots, \sigma_{D}$ denote a cosine sequence of $\Gamma$. Then (i), (ii) hold below.

(i) $\sigma_{i} \neq 0$ or $\sigma_{i+1} \neq 0 \quad(0 \leq i \leq D-1)$.

(ii) $\sigma_{D} \neq 0$.

Lemma 2.4 Let $\Gamma$ denote a bipartite distance-regular graph with diameter $D \geq 3$. Let $E, F$ denote primitive idempotents of $\Gamma$ with cosine sequences $\sigma_{0}, \sigma_{1}, \ldots, \sigma_{D}$ and $\rho_{0}, \rho_{1}, \ldots, \rho_{D}$, respectively. Then for all integers $i(1 \leq i \leq D-1)$,

$$
\sigma_{i+1} \rho_{i-1}-\sigma_{i-1} \rho_{i+1}=\sigma \sigma_{i}\left(\rho_{i-1}-\rho_{i+1}\right)-\rho \rho_{i}\left(\sigma_{i-1}-\sigma_{i+1}\right)
$$

Proof: Observe

$$
\left(\sigma \sigma_{i}-\sigma_{i+1}\right)\left(\rho_{i-1}-\rho_{i+1}\right)=\left(\rho \rho_{i}-\rho_{i+1}\right)\left(\sigma_{i-1}-\sigma_{i+1}\right)
$$

since both sides equal $c_{i}\left(\rho_{i-1}-\rho_{i+1}\right)\left(\sigma_{i-1}-\sigma_{i+1}\right) / k$ in view of (11). Multiplying out (14) and cancelling terms, we obtain (13).

Definition 2.5 Let $\Gamma$ denote a bipartite distance-regular graph. Let $E, F$ denote primitive idempotents of $\Gamma$, and let $\theta, \theta^{\prime}$ denote the corresponding eigenvalues. We say $E$ and $F$ are opposites whenever $\theta^{\prime}=-\theta$.

Lemma 2.6 Let $\Gamma$ denote a bipartite distance-regular graph with diameter $D \geq 3$. Let $E, F$ denote primitive idempotents of $\Gamma$ with cosine sequences $\sigma_{0}, \sigma_{1}, \ldots, \sigma_{D}$ and $\rho_{0}, \rho_{1}, \ldots, \rho_{D}$, respectively. Then the following are equivalent:

(i) $E$ and $F$ are opposites.

(ii) $\rho=-\sigma$.

(iii) $\rho_{i}=(-1)^{i} \sigma_{i}(0 \leq i \leq D)$.

Proof: $\quad$ Routine using Lemma 2.1.

Lemma 2.7 [2, Prop. 4.4.7] Let $\Gamma$ denote a bipartite distance-regular graph with diameter $D \geq 3$ and eigenvalues $\theta_{0}>\theta_{1}>\cdots>\theta_{D}$. Let $E$ denote a primitive idempotent of $\Gamma$ with cosine sequence $\sigma_{0}, \sigma_{1}, \ldots, \sigma_{D}$.

(i) Suppose $E=E_{0}$. Then $\sigma_{i}=1(0 \leq i \leq D)$.

(ii) Suppose $E=E_{D}$. Then $\sigma_{i}=(-1)^{i}(0 \leq i \leq D)$.

(iii) Suppose $E$ is one of $E_{1}, E_{2}, \ldots, E_{D-1}$. Then $-1<\sigma_{i}<1(1 \leq i \leq D-1)$. 
Definition 2.8 Let $\Gamma$ denote a bipartite distance-regular graph with valency $k$. We say that an eigenvalue $\theta$ of $\Gamma$ is trivial whenever $\theta=k$ or $\theta=-k$. We say that a primitive idempotent $E$ of $\Gamma$ is trivial whenever the associated eigenvalue is trivial. One can show that a primitive idempotent $E$ is trivial if and only if $\operatorname{rank}(E)=1$.

The following result appears in [18], although parts (i), (ii) of this result are from the folklore of the subject of distance-regular graphs.

Lemma 2.9 Let $\Gamma$ denote a bipartite distance-regular graph with diameter $D \geq 3$ and eigenvalues $\theta_{0}>\theta_{1}>\cdots>\theta_{D}$. Then (i)-(iii) hold below.

(i) $E_{0} \circ E_{i}=|X|^{-1} E_{i}(0 \leq i \leq D)$.

(ii) $E_{D} \circ E_{i}=|X|^{-1} E_{D-i}(0 \leq i \leq D)$.

(iii) Let $E, F$ denote primitive idempotents of $\Gamma$ other than $E_{0}$ and $E_{D}$. Then $E \circ F$ is not a scalar multiple of a primitive idempotent of $\Gamma$.

Lemma 2.10 Let $\Gamma$ denote a bipartite distance-regular graph with diameter $D \geq 3$ and eigenvalues $\theta_{0}>\theta_{1}>\cdots>\theta_{D}$. Then

$$
q_{i j}^{h}=q_{D-i, j}^{D-h}=q_{i, D-j}^{D-h}=q_{D-i, D-j}^{h} \quad(0 \leq h, i, j \leq D)
$$

Proof: To get the equation on the left, we take the entry-wise product of both sides of (8) with $E_{D}$ and apply Lemma 2.9(ii). The second equation follows since $q_{i j}^{h}=q_{j i}^{h}$ $(0 \leq h, i, j \leq D)$, and the third equation is a routine consequence of the first one.

Corollary 2.11 Let $\Gamma$ denote a bipartite distance-regular graph with diameter $D \geq 3$ and eigenvalues $\theta_{0}>\theta_{1}>\cdots>\theta_{D}$. Then

$$
q_{i j}^{D}=\delta_{i D-j} m_{i} \quad(0 \leq i, j \leq D) .
$$

Let $\Gamma$ denote a bipartite distance-regular graph with diameter $D \geq 4$ and valency $k \geq 3$. Let $E, F$ denote primitive idempotents of $\Gamma$. We mentioned the entry-wise product $E \circ F$ is nonzero, so $E \circ F$ is a linear combination of at least one primitive idempotent of $\Gamma$. Recall by Lemma 2.9 that $E \circ F$ is a scalar multiple of a single primitive idempotent of $\Gamma$ if and only if at least one of $E, F$ is trivial. Suppose $E, F$ are nontrivial, so $E \circ F$ is a linear combination of at least two distinct primitive idempotents of $\Gamma$. It is natural to consider when $E \circ F$ is a linear combination of two distinct primitive idempotents of $\Gamma$. This is the situation we consider in the following definition.

Definition 2.12 Let $\Gamma$ denote a bipartite distance-regular graph with diameter $D \geq 4$ and valency $k \geq 3$. We introduce a binary symmetric relation on the set of all primitive idempotents of $\Gamma$. We call this the taut relation. Let $E, F$ denote primitive idempotents of $\Gamma$. We say the pair $E, F$ is taut whenever (i) $E, F$ are nontrivial, and (ii) the entry-wise product $E \circ F$ is a linear combination of two distinct primitive idempotents of $\Gamma$. 
We now recall a class of bipartite distance-regular graphs that contain taut pairs of primitive idempotents.

Definition 2.13 Let $\Gamma$ denote a bipartite distance-regular graph with diameter $D \geq 3$, valency $k \geq 3$, and eigenvalues $\theta_{0}>\theta_{1}>\cdots>\theta_{D}$. Let $\theta$ denote a nontrivial eigenvalue of $\Gamma$. In [3], Curtin shows

$$
(\mu-1) \theta^{2} \leq(k-\mu)(k-2)
$$

and that the set of nontrivial eigenvalues $\theta$ of $\Gamma$ for which equality holds in (17) is either (i) empty, or (ii) $\left\{\theta_{1}, \theta_{D-1}\right\}$. $\Gamma$ is said to be 2-homogeneous if (ii) occurs.

Nomura obtains a classification of the 2-homogeneous bipartite distance-regular graphs in [14]. Let $\Gamma$ denote a bipartite distance-regular graph with diameter $D \geq 3$ and valency $k \geq 3$. Nomura shows that if $\Gamma$ is 2 -homogeneous and $D>5$, then $\Gamma$ is the D-cube. Furthermore, Curtin proves in [3] that the following are equivalent: (i) $\Gamma$ is 2-homogeneous, (ii) $\Gamma$ is an antipodal 2-cover and $Q$-polynomial, (iii) $\Gamma$ has a dual bipartite $Q$-polynomial structure.

Theorem 2.14 ([12]) Let $\Gamma$ denote a 2-homogeneous bipartite distance-regular graph with diameter $D \geq 4$, valency $k \geq 3$, and eigenvalues $\theta_{0}>\theta_{1}>\cdots>\theta_{D}$. Let $E, F$ denote nontrivial primitive idempotents of $\Gamma$. Then the pair $E, F$ is taut if and only if at least one of $E, F$ is equal to $E_{1}$ or $E_{D-1}$.

Definition 2.15 Let $\Gamma$ denote a bipartite distance-regular graph with diameter $D \geq 4$ and valency $k \geq 3$. We define $\Gamma$ to be taut whenever $\Gamma$ is not 2 -homogeneous and $\Gamma$ has at least one taut pair of primitive idempotents.

Taut distance-regular graphs with odd diameter and taut distance-regular graphs with even diameter appear to be fundamentally different objects, and we will handle them separately. In this paper, we will focus on taut distance-regular graphs with odd diameter. Of these, there are three known sporadic examples, each with diameter 5, and one infinite family. The sporadic examples are the Double Hoffman-Singleton graph [2, Section 13.1], Double Gewirtz graph [2, Section 11.4G], and Double 77-graph [2, p. 418]. The infinite family is given below.

Example 2.16 Given an integer $k \geq 3$, let $\Gamma$ denote the graph $2 . O_{k}$, the double cover of the Odd graph $O_{k}$ (see [2, Section 9.1D]). Recall $\Gamma$ is a bipartite distance-regular graph with valency $k$ and diameter $D=2 k-1$. The intersection numbers of $\Gamma$ are given by

$$
c_{2 i}=c_{2 i-1}=i \quad(1 \leq i \leq k-1) .
$$

The distinct eigenvalues of $\Gamma$ are $\pm 1, \pm 2, \ldots, \pm k$ [2, p. 414]. Moreover, the graph $\Gamma$ is taut. We will verify this in Example 5.14. 
Theorem 2.17 ([12]) Let $\Gamma$ denote a taut bipartite distance-regular graph with diameter $D \geq 4$, valency $k \geq 3$, and eigenvalues $\theta_{0}>\theta_{1}>\cdots>\theta_{D}$. Let $E, F$ denote nontrivial primitive idempotents of $\Gamma$.

(i) Suppose $D$ is odd, and let $d=(D-1) / 2$. Then the pair $E, F$ is taut if and only if the set $\{E, F\}$ is one of $\left\{E_{1}, E_{d}\right\},\left\{E_{1}, E_{d+1}\right\},\left\{E_{D-1}, E_{d}\right\},\left\{E_{D-1}, E_{d+1}\right\}$.

(ii) Suppose $D$ is even, and let $d=D / 2$. Then the pair $E, F$ is taut if and only if the set $\{E, F\}$ is one of $\left\{E_{1}, E_{d}\right\},\left\{E_{D-1}, E_{d}\right\}$.

We end this chapter with two results concerning 2-homogeneous bipartite distance-regular graphs.

Theorem 2.18 ([3]) Let $\Gamma$ denote a bipartite distance-regular graph with diameter $D \geq 3$, valency $k \geq 3$, and eigenvalues $\theta_{0}>\theta_{1}>\cdots>\theta_{D}$. Let $\theta$ denote a nontrivial eigenvalue of $\Gamma$, and let $\sigma_{0}, \sigma_{1}, \ldots, \sigma_{D}$ denote the associated cosine sequence. Then the following are equivalent:

(i) $\Gamma$ is 2-homogeneous and $\theta \in\left\{\theta_{1}, \theta_{D-1}\right\}$.

(ii) There exists a complex scalar $\lambda$ such that

$$
\sigma_{i-1}-\lambda \sigma_{i}+\sigma_{i+1}=0 \quad(1 \leq i \leq D-1) .
$$

(iii) There exists a complex scalar $\lambda$ such that

$$
\sigma_{i-1}-\lambda \sigma_{i}+\sigma_{i+1}=0 \quad(1 \leq i \leq 2) .
$$

Furthermore, suppose (i)-(iii) hold. Then $\lambda$ is real.

Lemma 2.19 ([3, 12]) Let $\Gamma$ denote a bipartite distance-regular graph with diameter $D \geq 4$ and valency $k \geq 3$. Set

$$
\Delta:=(k-2)\left(c_{3}-1\right)-(\mu-1) p_{22}^{2}
$$

Then $\Delta \geq 0$. Moreover, $\Gamma$ is 2-homogeneous if and only if $\Delta=0$ and $\Gamma$ has at least one taut pair of primitive idempotents.

\section{The Christoffel-Darboux formula}

Lemma 3.1 (Christoffel-Darboux formula) [1, Theorem III.1.3] Let $\Gamma$ denote a distance-regular graph with diameter $D \geq 3$. Let $\sigma_{0}, \sigma_{1}, \ldots, \sigma_{D}$ and $\rho_{0}, \rho_{1}, \ldots, \rho_{D}$ denote cosine sequences of $\Gamma$. Then

$$
k(\sigma-\rho) \sum_{h=0}^{i} k_{h} \sigma_{h} \rho_{h}=k_{i} b_{i}\left(\sigma_{i+1} \rho_{i}-\sigma_{i} \rho_{i+1}\right) \quad(0 \leq i \leq D),
$$

where $\sigma_{D+1}, \rho_{D+1}$ are indeterminates. 
Corollary 3.2 Let $\Gamma$ denote a distance-regular graph with diameter $D \geq 3$. Let $\sigma_{0}, \sigma_{1}, \ldots$, $\sigma_{D}$ denote a cosine sequence of $\Gamma$. Then

$$
k(\sigma-1) \sum_{h=0}^{i} k_{h} \sigma_{h}=k_{i} b_{i}\left(\sigma_{i+1}-\sigma_{i}\right) \quad(0 \leq i \leq D),
$$

where $\sigma_{D+1}$ is indeterminate.

Proof: By Lemma 2.7(i), the cosine sequence associated with $E_{0}$ is $1,1, \ldots, 1$. Let $\rho_{j}=$ $1(0 \leq j \leq D)$ in (22) to obtain the desired result.

We now obtain an equation similar in form to the Christoffel-Darboux formula; this equation is for bipartite distance-regular graphs.

Lemma 3.3 Let $\Gamma$ denote a bipartite distance-regular graph with diameter $D \geq 4$. Let $\sigma_{0}, \sigma_{1}, \ldots, \sigma_{D}$ and $\rho_{0}, \rho_{1}, \ldots, \rho_{D}$ denote cosine sequences of $\Gamma$. For $0 \leq i \leq D$,

$$
k^{2}\left(\sigma^{2}-\rho^{2}\right) \sum_{\substack{0 \leq h \leq i-1 \\ i-h}} k_{h} \sigma_{h} \rho_{h}=k_{i} c_{i} b_{i}\left(\sigma_{i+1} \rho_{i-1}-\sigma_{i-1} \rho_{i+1}\right),
$$

where $\sigma_{-1}, \rho_{-1}, \sigma_{D+1}, \rho_{D+1}$ are indeterminates.

Proof: Repeatedly applying (11) and using the fact that $b_{j}=k-c_{j}(0 \leq j \leq D)$, we find that for $0 \leq h \leq D-1$,

$$
k^{2} \sigma^{2} \sigma_{h}=c_{h} c_{h-1} \sigma_{h-2}+\left(c_{h} b_{h-1}+b_{h} c_{h+1}\right) \sigma_{h}+b_{h} b_{h+1} \sigma_{h+2},
$$

where we define $c_{-1}:=b_{-1}:=0$, and where $\sigma_{-2}$ is indeterminate. Similarly,

$$
k^{2} \rho^{2} \rho_{h}=c_{h} c_{h-1} \rho_{h-2}+\left(c_{h} b_{h-1}+b_{h} c_{h+1}\right) \rho_{h}+b_{h} b_{h+1} \rho_{h+2},
$$

where $\rho_{-2}$ is indeterminate.

Subtracting $k_{h} \sigma_{h}$ times (26) from $k_{h} \rho_{h}$ times (25) and using (4), we find

$$
\begin{aligned}
k^{2}\left(\sigma^{2}-\rho^{2}\right) k_{h} \sigma_{h} \rho_{h}= & k_{h+1} c_{h+1} b_{h+1}\left(\sigma_{h+2} \rho_{h}-\sigma_{h} \rho_{h+2}\right) \\
& -k_{h-1} c_{h-1} b_{h-1}\left(\sigma_{h} \rho_{h-2}-\sigma_{h-2} \rho_{h}\right)
\end{aligned}
$$

for $0 \leq h \leq D-1$, where we define $k_{-1}:=0$. Fix an integer $i(0 \leq i \leq D)$. Summing (27) over all $h$ such that $0 \leq h \leq i-1$ and such that $i-h$ is odd, we obtain (24).

Corollary 3.4 Let $\Gamma$ denote a bipartite distance-regular graph with diameter $D \geq 4$. Let $\sigma_{0}, \sigma_{1}, \ldots, \sigma_{D}$ denote a cosine sequence of $\Gamma$. For $0 \leq i \leq D$,

$$
k^{2}\left(\sigma^{2}-1\right) \sum_{\substack{0 \leq h \leq i-1 \\ i-h \text { odd }}} k_{h} \sigma_{h}=k_{i} c_{i} b_{i}\left(\sigma_{i+1}-\sigma_{i-1}\right),
$$

where $\sigma_{-1}, \sigma_{D+1}$ are indeterminates. 
Proof: By Lemma 2.7(i), the cosine sequence associated with $E_{0}$ is $1,1, \ldots, 1$. Letting $\rho_{j}=1(0 \leq j \leq D)$ in $(24)$, we obtain the desired result.

\section{Some equations involving cosine sequences}

Definition 4.1 Throughout this section, we let $\Gamma$ denote a bipartite distance-regular graph with diameter $D \geq 4$ and valency $k \geq 3$. Let $\theta_{0}>\theta_{1}>\cdots>\theta_{D}$ denote the distinct eigenvalues of $\Gamma$. Furthermore, we let $E$ and $F$ denote nontrivial primitive idempotents of $\Gamma$. We let $\sigma_{0}, \sigma_{1}, \ldots, \sigma_{D}$ and $\rho_{0}, \rho_{1}, \ldots, \rho_{D}$ denote the corresponding cosine sequences. We let $G$ and $H$ denote distinct primitive idempotents of $\Gamma$ with cosine sequences $\gamma_{0}, \gamma_{1}, \ldots, \gamma_{D}$ and $\epsilon_{0}, \epsilon_{1}, \ldots, \epsilon_{D}$, respectively.

Lemma 4.2 With reference to Definition 4.1, the following are equivalent:

(i) $E \circ F \in \operatorname{span}\{G, H\}$.

(ii) There exist complex scalars $a, b$ such that for $0 \leq i \leq D$,

$$
\sigma_{i} \rho_{i}=a \gamma_{i}+b \epsilon_{i}
$$

Suppose (i), (ii) hold. Then $a, b$ are given by

$$
a=\frac{\sigma \rho-\epsilon}{\gamma-\epsilon}, \quad b=\frac{\gamma-\sigma \rho}{\gamma-\epsilon} .
$$

Moreover, $a, b$ are nonzero and real.

Proof: Let $m_{\sigma}, m_{\rho}, m_{\gamma}, m_{\epsilon}$ denote the multiplicities of $E, F, G, H$, respectively. Recall

$$
\begin{aligned}
E & =|X|^{-1} m_{\sigma} \sum_{i=0}^{D} \sigma_{i} A_{i}, & F & =|X|^{-1} m_{\rho} \sum_{i=0}^{D} \rho_{i} A_{i}, \\
G & =|X|^{-1} m_{\gamma} \sum_{i=0}^{D} \gamma_{i} A_{i}, & H & =|X|^{-1} m_{\epsilon} \sum_{i=0}^{D} \epsilon_{i} A_{i} .
\end{aligned}
$$

(i) $\Rightarrow$ (ii) By assumption, there exist complex scalars $\psi, \phi$ such that

$$
E \circ F=\psi G+\phi H
$$

Eliminating $E, F, G, H$ in (33) using (31), (32), and evaluating the result using (7), we obtain

$$
m_{\sigma} m_{\rho} \sigma_{i} \rho_{i}=|X|\left(\psi m_{\gamma} \gamma_{i}+\phi m_{\epsilon} \epsilon_{i}\right) \quad(0 \leq i \leq D)
$$

Apparently (29) holds with

$$
a=\frac{|X| \psi m_{\gamma}}{m_{\sigma} m_{\rho}}, \quad b=\frac{|X| \phi m_{\epsilon}}{m_{\sigma} m_{\rho}} .
$$


(ii) $\Rightarrow$ (i) By (31), (7), (29), and (32), we have

$$
\begin{aligned}
E \circ F & =|X|^{-2} m_{\sigma} m_{\rho} \sum_{i=0}^{D} \sigma_{i} \rho_{i} A_{i} \\
& =|X|^{-2} m_{\sigma} m_{\rho}\left(a \sum_{i=0}^{D} \gamma_{i} A_{i}+b \sum_{i=0}^{D} \epsilon_{i} A_{i}\right) \\
& =|X|^{-1} m_{\sigma} m_{\rho}\left(\frac{a}{m_{\gamma}} G+\frac{b}{m_{\epsilon}} H\right),
\end{aligned}
$$

proving (i).

Now suppose (i), (ii) hold. To obtain (30), set $i=0,1$ in (29) and solve the resulting linear equations for $a$ and $b$. To show $a, b$ are real and nonzero, we refer to the proof of (i) $\Rightarrow$ (ii) above. One may show $\psi, \phi$ are real and nonzero using Lemma 2.9(iii) and the fact that Krein parameters are real. Now $a, b$ are real and nonzero by (34).

With reference to Definition 4.1, suppose for the moment that $E, F, G, H$ satisfy (i), (ii) in Lemma 4.2. In the following lemma, we consider the case when one of $G, H$ is trivial.

Lemma 4.3 With reference to Definition 4.1, suppose (i), (ii) hold in Lemma 4.2. Then

(i) $E=F$ if and only if one of $G, H$ is equal to $E_{0}$.

(ii) $E, F$ are opposites if and only if one of $G, H$ is equal to $E_{D}$.

Furthermore, suppose $E=F$. Then $\Gamma$ is 2-homogeneous, and $E \in\left\{E_{1}, E_{D-1}\right\}$. Now suppose $E, F$ are opposites. Then $\Gamma$ is 2-homogeneous, and $E, F$ is a permutation of $E_{1}, E_{D-1}$.

\section{Proof:}

(i) Routine consequence of (8), (9), and the linear independence of the primitive idempotents.

(ii) By Definition 2.5 and (10), $E, F$ are opposites if and only if there exists an integer $i$ $(0 \leq i \leq D)$ such that $E=E_{i}, F=E_{D-i}$. The result is now a routine consequence of (8), (16), and the linear independence of the primitive idempotents.

Now suppose (i) or (ii) holds. By Lemma 4.2(i) and since $E, F$ are nontrivial, we find the pair $E, F$ is taut. Observe $\Gamma$ is not taut since Theorem 2.17(i), (ii) do not hold; thus $\Gamma$ is 2-homogeneous by Definition 2.15. If (i) holds, then $E \in\left\{E_{1}, E_{D-1}\right\}$ by Theorem 2.14. If (ii) holds, then $E, F$ is a permutation of $E_{1}, E_{D-1}$ by Theorem 2.14 .

Lemma 4.4 With reference to Definition 4.1, suppose (i), (ii) hold in Lemma 4.2 and that $E, F$ are distinct. Then for any integer $i(0 \leq i \leq D-1)$,

$$
\frac{\sigma_{i+1} \rho_{i}-\sigma_{i} \rho_{i+1}}{\sigma-\rho}=a \frac{\gamma_{i+1}-\gamma_{i}}{\gamma-1}+b \frac{\epsilon_{i+1}-\epsilon_{i}}{\epsilon-1}
$$


where $a, b$ are from (30). Observe the denominators in (35) are nonzero by Lemma 2.7 and Lemma 4.3.

Proof: Using (22), (29), and (23), we observe

$$
\begin{aligned}
\frac{k_{i} b_{i}}{k} \frac{\sigma_{i+1} \rho_{i}-\sigma_{i} \rho_{i+1}}{\sigma-\rho} & =\sum_{h=0}^{i} k_{h} \sigma_{h} \rho_{h} \\
& =a \sum_{h=0}^{i} k_{h} \gamma_{h}+b \sum_{h=0}^{i} k_{h} \epsilon_{h} \\
& =\frac{k_{i} b_{i}}{k}\left(a \frac{\gamma_{i+1}-\gamma_{i}}{\gamma-1}+b \frac{\epsilon_{i+1}-\epsilon_{i}}{\epsilon-1}\right),
\end{aligned}
$$

and the result follows.

Lemma 4.5 With reference to Definition 4.1, suppose (i), (ii) hold in Lemma 4.2 and that $E, F$ are neither equal nor opposites. Then for any integer $i \quad(1 \leq i \leq D-1)$,

$$
\frac{\sigma_{i+1} \rho_{i-1}-\sigma_{i-1} \rho_{i+1}}{\sigma^{2}-\rho^{2}}=a \frac{\gamma_{i+1}-\gamma_{i-1}}{\gamma^{2}-1}+b \frac{\epsilon_{i+1}-\epsilon_{i-1}}{\epsilon^{2}-1}
$$

where $a, b$ are from (30). Observe the denominators in (36) are nonzero by Lemma 2.6, Lemma 2.7, and Lemma 4.3.

Proof: By (24), (29), and (28), we observe

$$
\begin{aligned}
\frac{k_{i} c_{i} b_{i}}{k^{2}} \frac{\sigma_{i+1} \rho_{i-1}-\sigma_{i-1} \rho_{i+1}}{\sigma^{2}-\rho^{2}} & =\sum_{\substack{0 \leq h \leq i-1 \\
i-h_{\text {odd }}}} k_{h} \sigma_{h} \rho_{h} \\
& =a \sum_{\substack{0 \leq h \leq i-1 \\
i-h_{\text {odd }}}} k_{h} \gamma_{h}+b \sum_{\substack{0 \leq h \leq i-1 \\
i-h_{\text {odd }}}} k_{h} \epsilon_{h} \\
& =\frac{k_{i} c_{i} b_{i}}{k^{2}}\left(a \frac{\gamma_{i+1}-\gamma_{i-1}}{\gamma^{2}-1}+b \frac{\epsilon_{i+1}-\epsilon_{i-1}}{\epsilon^{2}-1}\right),
\end{aligned}
$$

and the result follows.

\section{The main results}

In the following theorem, we consider the equation

$$
\sigma_{i+1} \rho_{i+1}-\sigma_{i-1} \rho_{i-1}=\alpha \sigma_{i}\left(\rho_{i+1}-\rho_{i-1}\right)+\beta \rho_{i}\left(\sigma_{i+1}-\sigma_{i-1}\right)
$$


Theorem 5.1 Let $\Gamma$ denote a bipartite distance-regular graph with diameter $D \geq 4$ and valency $k \geq 3$. Let $E$ and $F$ denote nontrivial primitive idempotents of $\Gamma$ with cosine sequences $\sigma_{0}, \sigma_{1}, \ldots, \sigma_{D}$ and $\rho_{0}, \rho_{1}, \ldots, \rho_{D}$, respectively. Then the following are equivalent:

(i) $E, F$ is a taut pair.

(ii) There exist complex scalars $\alpha, \beta$ such that equality holds in (37) for all integers $i(1 \leq i \leq D-1)$.

(iii) There exist complex scalars $\alpha, \beta$ such that equality holds in (37) for $i=1,2,3$.

Proof: Let $\theta_{0}>\theta_{1}>\cdots>\theta_{D}$ denote the distinct eigenvalues of $\Gamma$.

(i) $\Rightarrow$ (ii) First assume $E=F$, so $\rho_{j}=\sigma_{j}$ for $0 \leq j \leq D$. By Lemma 4.3, we find $\Gamma$ is 2-homogeneous, and that $E$ is one of $E_{1}, E_{D-1}$. By Theorem 2.18, there exists a complex scalar $\lambda$ such that

$$
\sigma_{i+1}+\sigma_{i-1}=\lambda \sigma_{i} \quad(1 \leq i \leq D-1)
$$

Using (38) and the fact that $\rho_{j}=\sigma_{j}(0 \leq j \leq D)$, we find that if we set $\alpha=\lambda, \beta=0$, then (37) holds for $1 \leq i \leq D-1$.

Now assume $E, F$ are opposites. By Lemma 2.6, $\rho_{j}=(-1)^{j} \sigma_{j}$ for $0 \leq j \leq D$. Applying Lemma 4.3 , we find $\Gamma$ is 2-homogeneous, and $E, F$ is a permutation of $E_{1}, E_{D-1}$. By Theorem 2.18 , there exists a complex scalar $\lambda$ such that

$$
\sigma_{i+1}+\sigma_{i-1}=\lambda \sigma_{i} \quad(1 \leq i \leq D-1)
$$

Using (39) and the fact that $\rho_{j}=(-1)^{j} \sigma_{j}(0 \leq j \leq D)$, we find that if we set $\alpha=\lambda, \beta=0$, then (37) holds for $1 \leq i \leq D-1$.

Now assume $E$ and $F$ are not equal nor opposites. By assumption $E \circ F$ is a linear combination of two distinct primitive idempotents, which we denote by $G, H$. Let $\gamma_{0}, \gamma_{1}, \ldots, \gamma_{D}$ and $\epsilon_{0}, \epsilon_{1}, \ldots, \epsilon_{D}$ denote the cosine sequences for $G$ and $H$, respectively. To obtain (37), we will combine (13), (29), (35), and (36) as follows. Fix an integer $i(1 \leq i \leq D-1)$. By (29),

$$
\sigma_{i+1} \rho_{i+1}-\sigma_{i-1} \rho_{i-1}=a\left(\gamma_{i+1}-\gamma_{i-1}\right)+b\left(\epsilon_{i+1}-\epsilon_{i-1}\right)
$$

where $a, b$ are from (30). Adding (35) at $i$ to (35) at $i-1$, we obtain

$$
\frac{\sigma_{i}\left(\rho_{i-1}-\rho_{i+1}\right)}{\sigma-\rho}-\frac{\rho_{i}\left(\sigma_{i-1}-\sigma_{i+1}\right)}{\sigma-\rho}=\frac{a\left(\gamma_{i+1}-\gamma_{i-1}\right)}{\gamma-1}+\frac{b\left(\epsilon_{i+1}-\epsilon_{i-1}\right)}{\epsilon-1} .
$$

Evaluating the left-hand side of (36) using (13), we find

$$
\frac{\sigma \sigma_{i}\left(\rho_{i-1}-\rho_{i+1}\right)}{\sigma^{2}-\rho^{2}}-\frac{\rho \rho_{i}\left(\sigma_{i-1}-\sigma_{i+1}\right)}{\sigma^{2}-\rho^{2}}=\frac{a\left(\gamma_{i+1}-\gamma_{i-1}\right)}{\gamma^{2}-1}+\frac{b\left(\epsilon_{i+1}-\epsilon_{i-1}\right)}{\epsilon^{2}-1} .
$$

Consider the equation Eq which is (40) minus the product of $u$ with (41) plus the product of $v$ with (42), where

$$
u=\epsilon+\gamma, \quad v=(1+\epsilon)(1+\gamma) .
$$


In the equation Eq, the right-hand side is 0 , since

$$
1-\frac{u}{\gamma-1}+\frac{v}{\gamma^{2}-1}=0, \quad 1-\frac{u}{\epsilon-1}+\frac{v}{\epsilon^{2}-1}=0 .
$$

Evaluating the left-hand side of the equation Eq, we obtain (37), where

$$
\alpha=\frac{u}{\rho-\sigma}-\frac{v \sigma}{\rho^{2}-\sigma^{2}}, \quad \beta=\frac{u}{\sigma-\rho}-\frac{v \rho}{\sigma^{2}-\rho^{2}} .
$$

(ii) $\Rightarrow$ (iii) Immediate.

(iii) $\Rightarrow$ (i) First assume $\rho \neq \pm \sigma$. Consider the matrix

$$
B:=\left(\begin{array}{ccc}
\sigma_{2} \rho_{2}-1 & \sigma\left(\rho_{2}-1\right) & \rho\left(\sigma_{2}-1\right) \\
\sigma_{3} \rho_{3}-\sigma \rho & \sigma_{2}\left(\rho_{3}-\rho\right) & \rho_{2}\left(\sigma_{3}-\sigma\right) \\
\sigma_{4} \rho_{4}-\sigma_{2} \rho_{2} & \sigma_{3}\left(\rho_{4}-\rho_{2}\right) & \rho_{3}\left(\sigma_{4}-\sigma_{2}\right)
\end{array}\right) .
$$

On one hand, $B$ is singular, so $\operatorname{det}(B)=0$. On the other hand, recursively eliminating $\sigma_{2}, \sigma_{3}, \sigma_{4}, \rho_{2}, \rho_{3}, \rho_{4}$ in the entries of $B$ using (12), one may verify that $\operatorname{det}(B)$ is equal to

$$
k^{5} b_{1}^{-6} b_{2}^{-4} b_{3}^{-2}\left(\sigma^{2}-1\right)^{2}\left(\rho^{2}-1\right)^{2}\left(\rho^{2}-\sigma^{2}\right)
$$

times

$$
f \sigma^{2} \rho^{2}+g\left(\sigma^{2}+\rho^{2}\right)+h
$$

where

$$
\begin{aligned}
f & =k^{4} \mu\left(\Delta+c_{3}-\mu\right), \\
g & =-k^{2} b_{2}^{2}\left(c_{3}-1\right), \\
h & =b_{2}^{2}\left(b_{2}\left(c_{3}-1\right)+\mu b_{3}(k-2)\right),
\end{aligned}
$$

and where $\Delta$ is from (21). (To do this, we must use the fact that $p_{22}^{2}=\mu^{-1}\left(b_{2}\left(c_{3}-1\right)+\right.$ $\mu(k-2))$ [2, Lemma 4.1.7]). Observe factor (43) is not zero, since we assume $\sigma^{2} \neq \rho^{2}$ and since $\sigma^{2} \neq 1, \rho^{2} \neq 1$ by Lemma 2.7(iii). Apparently factor (44) is zero. Applying [12, Corollary 3.11], we find $E, F$ is a taut pair.

Now assume $\rho= \pm \sigma$. If $\rho=\sigma$, then $\rho_{j}=\sigma_{j}$ for $0 \leq j \leq D$. If $\rho=-\sigma$, then $\rho_{j}=(-1)^{j} \sigma_{j}$ for $0 \leq j \leq D$ by Lemma 2.6. We assume (37) holds for $1 \leq i \leq 3$; evaluating these equations using $\rho_{j}=\sigma_{j}$ or $\rho_{j}=(-1)^{j} \sigma_{j}$ as appropriate, we find

$$
\sigma_{i+1}^{2}-\sigma_{i-1}^{2}=\lambda \sigma_{i}\left(\sigma_{i+1}-\sigma_{i-1}\right) \quad(1 \leq i \leq 3),
$$

where $\lambda=\alpha+\beta$ if $\rho=\sigma$ and $\lambda=\alpha-\beta$ if $\rho=-\sigma$. Letting $i=1,2$ in (45), we obtain

$$
\begin{aligned}
\sigma_{2}^{2}-1 & =\lambda \sigma\left(\sigma_{2}-1\right), \\
\sigma_{3}^{2}-\sigma^{2} & =\lambda \sigma_{2}\left(\sigma_{3}-\sigma\right) .
\end{aligned}
$$


Observe $\sigma_{2} \neq \pm 1$ by Lemma 2.7(iii). Dividing both sides of (46) by $\sigma_{2}-1$, we obtain

$$
\sigma_{2}+1=\lambda \sigma
$$

Since $\sigma_{2} \neq-1$, we see $\sigma \neq 0$ in view of (48). We mentioned $\sigma_{2} \neq 1$, so $\sigma_{3} \neq \sigma$ by (12). Dividing both sides of (47) by $\sigma_{3}-\sigma$, we obtain

$$
\sigma_{3}+\sigma=\lambda \sigma_{2}
$$

By (48) and (49), we find condition Theorem 2.18(iii) holds. Applying this theorem, we find $\Gamma$ is 2-homogeneous and $E \in\left\{E_{1}, E_{D-1}\right\}$. Now $E, F$ is a taut pair by Theorem 2.14.

Let $\Gamma$ denote a bipartite distance-regular graph with diameter $D \geq 4$ and valency $k \geq 3$. Assume $\Gamma$ has at least one taut pair of primitive idempotents. By Definition 2.15, one of the following holds: (i) $\Gamma$ is 2-homogeneous, (ii) $\Gamma$ is taut and $D$ is even, (iii) $\Gamma$ is taut and $D$ is odd. Case (i) has already been explored by Curtin in [3] and Nomura in [14], so we will not discuss it further here. Cases (ii) and (iii) appear to be fundamentally different, and we will handle them separately. For the rest of this paper, we will focus on case (iii).

Let $\Gamma$ denote a taut bipartite distance-regular graph with odd diameter $D \geq 5$ and valency $k \geq 3$. Let $E, F$ denote a taut pair of primitive idempotents of $\Gamma$. Let $\theta, \theta^{\prime}$ denote the corresponding eigenvalues, and let $\sigma_{0}, \sigma_{1}, \ldots, \sigma_{D}$ and $\rho_{0}, \rho_{1}, \ldots, \rho_{D}$ denote the corresponding cosine sequences. Let $\alpha, \beta$ denote complex scalars satisfying (37) for $1 \leq i \leq D-1$. We use (37) to solve for $\alpha, \beta$ in terms of $k, \mu, \sigma, \rho$. Since $\sigma=\theta / k, \rho=\theta^{\prime} / k$, this gives $\alpha, \beta$ in terms of $k, \mu, \theta, \theta^{\prime}$. Our result is the following.

Corollary 5.2 Let $\Gamma$ denote a taut bipartite distance-regular graph with odd diameter $D \geq 5$ and valency $k \geq 3$. Let $E, F$ denote a taut pair of primitive idempotents of $\Gamma$. Let $\theta, \theta^{\prime}$ denote the corresponding eigenvalues, and let $\sigma_{0}, \sigma_{1}, \ldots, \sigma_{D}$ and $\rho_{0}, \rho_{1}, \ldots, \rho_{D}$ denote the corresponding cosine sequences. Let $\alpha, \beta$ denote complex scalars satisfying (37) for $1 \leq i \leq D-1$. Then

$$
\begin{aligned}
& \alpha=\frac{\theta}{k}+\frac{\theta\left(k^{2}-\theta^{2}\right)\left(b_{2}(k-2)-\theta^{\prime 2}(\mu-1)\right)}{k\left(\theta^{2}-\theta^{\prime 2}\right) b_{1} b_{2}}, \\
& \beta=\frac{\theta^{\prime}}{k}+\frac{\theta^{\prime}\left(k^{2}-\theta^{\prime 2}\right)\left(b_{2}(k-2)-\theta^{2}(\mu-1)\right)}{k\left(\theta^{\prime 2}-\theta^{2}\right) b_{1} b_{2}} .
\end{aligned}
$$

In particular, $\alpha, \beta$ are real and are uniquely determined by $k, \mu, \theta, \theta^{\prime}$.

Proof: Setting $i=1,2$ in (37), we obtain the equations

$$
\begin{aligned}
\sigma_{2} \rho_{2}-1 & =\alpha \sigma\left(\rho_{2}-1\right)+\beta \rho\left(\sigma_{2}-1\right), \\
\sigma_{3} \rho_{3}-\sigma \rho & =\alpha \sigma_{2}\left(\rho_{3}-\rho\right)+\beta \rho_{2}\left(\sigma_{3}-\sigma\right),
\end{aligned}
$$


where $\sigma=\sigma_{1}, \rho=\rho_{1}$. Observe these equations are linear in $\alpha, \beta$. Recursively eliminating $\sigma_{2}, \sigma_{3}, \rho_{2}, \rho_{3}$ in these equations using (12) and recalling $\sigma=\theta / k, \rho=\theta^{\prime} / k$, we routinely find that the system (52), (53) has a unique solution for $\alpha, \beta$ which satisfies (50), (51).

Definition 5.3 Let $\Gamma$ denote a taut bipartite distance-regular graph with odd diameter $D \geq 5$, valency $k \geq 3$, and eigenvalues $\theta_{0}>\theta_{1}>\cdots>\theta_{D}$. Let $d=(D-1) / 2$, and consider the set consisting of the following four eigenvalues:

$$
\theta_{1}, \quad \theta_{d}, \quad \theta_{d+1}, \quad \theta_{D-1} .
$$

Recall $\theta_{D-1}=-\theta_{1}$ and $\theta_{d+1}=-\theta_{d}$. Let $\theta$ denote an eigenvalue in (54), and let $E$ denote the corresponding primitive idempotent. By the weight of $\theta$ (or $E$ ), we mean the real scalar

$$
\frac{\theta}{k}+\frac{\theta\left(k^{2}-\theta^{2}\right)\left(b_{2}(k-2)-\theta^{\prime 2}(\mu-1)\right)}{k\left(\theta^{2}-\theta^{\prime 2}\right) b_{1} b_{2}},
$$

where $\theta^{\prime}$ is any eigenvalue in (54) other than $\theta,-\theta$.

We introduced the notion of a weight in Definition 5.3 so that we may easily discuss the scalars $\alpha, \beta$ appearing in (37). To clarify how we use this notation, we give the following corollary.

Corollary 5.4 Let $\Gamma$ denote a taut bipartite distance-regular graph with odd diameter $D \geq 5$ and valency $k \geq 3$. Let $E, F$ denote a taut pair of primitive idempotents with cosine sequences $\sigma_{0}, \sigma_{1}, \ldots, \sigma_{D}$ and $\rho_{0}, \rho_{1}, \ldots, \rho_{D}$, respectively. Let $\alpha, \beta$ denote complex scalars. Then the following are equivalent:

(i) The scalars $\alpha, \beta$ satisfy (37) for $1 \leq i \leq D-1$.

(ii) The scalars $\alpha, \beta$ are the weights of $E, \bar{F}$, respectively.

Proof: Immediate using Theorem 2.17(i), Corollary 5.2, and Definition 5.3.

Lemma 5.5 Let $\Gamma$ denote a taut bipartite distance-regular graph with odd diameter $D \geq$ 5 , valency $k \geq 3$, and eigenvalues $\theta_{0}>\theta_{1}>\cdots>\theta_{D}$. Let $d=(D-1) / 2$, and let $E$ denote one of $E_{1}, E_{d}, E_{d+1}, E_{D-1}$. Let $\alpha$ denote the weight of $E$, and let $\sigma$ denote the first cosine of $E$. Then (i), (ii) hold below.

(i) Suppose $E=E_{1}$ or $E=E_{d+1}$. Then $\alpha>\sigma$.

(ii) Suppose $E=E_{d}$ or $E=E_{D-1}$. Then $\alpha<\sigma$.

Proof: Let $\theta$ denote the eigenvalue associated with $E$, and let $\theta^{\prime}$ denote one of $\theta_{1}, \theta_{d}, \theta_{d+1}$, $\theta_{D-1}$ not equal to $\theta$ or $-\theta$. Recalling $\theta=k \sigma$ and using Definition 5.3, we find $\alpha-\sigma$ equals

$$
\left(k^{2}-\theta^{2}\right)\left(b_{2}(k-2)-\theta^{\prime 2}(\mu-1)\right) k^{-1} b_{1}^{-1} b_{2}^{-1}
$$

times $\theta\left(\theta^{2}-\theta^{\prime 2}\right)^{-1}$. In (56) the factor on the left is positive since $\theta<k$. The factor in the middle is positive by (17) and since $\Gamma$ is not 2 -homogeneous. The remaining factors in 
(56) are also positive, so (56) is positive. Using $\theta_{1}>\theta_{d}>\theta_{d+1}>\theta_{D-1}$ and $\theta_{1}=-\theta_{D-1}$, $\theta_{d}=-\theta_{d+1}$, we find $\theta\left(\theta^{2}-\theta^{\prime 2}\right)^{-1}$ is positive in case (i) and negative in case (ii). The result follows.

Lemma 5.6 Let $\Gamma$ denote a taut bipartite distance-regular graph with odd diameter $D \geq$ 5 , valency $k \geq 3$, and eigenvalues $\theta_{0}>\theta_{1}>\cdots>\theta_{D}$. Let $d=(D-1) / 2$, and let $\sigma_{0}, \sigma_{1}, \ldots, \sigma_{D}$ denote the cosine sequence associated with any one of $\theta_{1}, \theta_{d}, \theta_{d+1}, \theta_{D-1}$. Then the expressions

$$
\sigma_{i+1}-\sigma_{i-1}, \quad \sigma \sigma_{i}-\sigma_{i-1}, \quad \sigma \sigma_{i}-\sigma_{i+1}
$$

are nonzero for $1 \leq i \leq D-1$.

Proof: By Corollary 2.2, it suffices to show $\sigma_{i+1}-\sigma_{i-1}$ is nonzero. Let $\theta$ denote the eigenvalue associated with the cosine sequence $\sigma_{0}, \sigma_{1}, \ldots, \sigma_{D}$. The result is true for $\theta=\theta_{1}$ and $\theta=\theta_{D-1}$ by (6) and Lemma 2.6. Now assume $\theta$ is one of $\theta_{d}, \theta_{d+1}$. Fix an integer $i(1 \leq$ $i \leq D-1)$ and assume $\sigma_{i+1}=\sigma_{i-1}$. We obtain a contradiction. To do this, we first observe by Corollary 2.2 that $\sigma_{i-1}, \sigma_{i+1}, \sigma \sigma_{i}$ are equal. Let $E$ denote the primitive idempotent of $\Gamma$ associated with $\theta$. Let $F$ denote the primitive idempotent of $\Gamma$ associated with $\theta_{1}$, and let $\rho_{0}, \rho_{1}, \ldots, \rho_{D}$ denote the corresponding cosine sequence. By Theorem 2.17(i), $E, F$ is a taut pair. Applying Corollary 5.4, we find

$$
\sigma_{i+1} \rho_{i+1}-\sigma_{i-1} \rho_{i-1}=\alpha \sigma_{i}\left(\rho_{i+1}-\rho_{i-1}\right)+\beta \rho_{i}\left(\sigma_{i+1}-\sigma_{i-1}\right)
$$

where $\alpha, \beta$ denote the weights of $E, F$, respectively. Setting $\sigma_{i+1}=\sigma \sigma_{i}, \sigma_{i-1}=\sigma \sigma_{i}$ in (57), we find

$$
(\sigma-\alpha) \sigma_{i}\left(\rho_{i+1}-\rho_{i-1}\right)=0
$$

If $\sigma_{i}=0$ then $\sigma_{i+1}=0$, violating Corollary 2.3(i); thus $\sigma_{i} \neq 0$. Recall $\rho_{0}, \rho_{1}, \ldots, \rho_{D}$ is the cosine sequence for $\theta_{1}$, so $\rho_{i+1} \neq \rho_{i-1}$ by (6). Thus $\sigma=\alpha$, contradicting Lemma 5.5. We conclude $\sigma_{i+1}-\sigma_{i-1}$ is nonzero, as desired.

Theorem 5.7 Let $\Gamma$ denote a taut bipartite distance-regular graph with odd diameter $D \geq$ 5 and valency $k \geq 3$. Let $E, F$ denote a taut pair of primitive idempotents. Let $\sigma_{0}, \sigma_{1}, \ldots, \sigma_{D}$ and $\rho_{0}, \rho_{1}, \ldots, \rho_{D}$ denote the corresponding cosine sequences, respectively, and let $\alpha, \beta$ denote the corresponding weights. Then

$$
\begin{gathered}
\frac{\sigma_{i+1}-\alpha \sigma_{i}}{\sigma \sigma_{i}-\sigma_{i-1}}=\frac{\beta \rho_{i}-\rho_{i-1}}{\rho \rho_{i}-\rho_{i-1}} \quad(1 \leq i \leq D-1), \\
\frac{\rho_{i+1}-\beta \rho_{i}}{\rho \rho_{i}-\rho_{i-1}}=\frac{\alpha \sigma_{i}-\sigma_{i-1}}{\sigma \sigma_{i}-\sigma_{i-1}} \quad(1 \leq i \leq D-1) .
\end{gathered}
$$

Observe the denominators in (59), (60) are nonzero by Lemma 5.6. 
Proof: Multiplying (37) by $\sigma \sigma_{i}-\sigma_{i-1}$, multiplying (13) by $\sigma_{i+1}-\alpha \sigma_{i}$, and taking the difference between the two products, we find $\sigma_{i-1}-\sigma_{i+1}$ times

$$
\left(\sigma_{i+1}-\alpha \sigma_{i}\right)\left(\rho \rho_{i}-\rho_{i-1}\right)-\left(\beta \rho_{i}-\rho_{i-1}\right)\left(\sigma \sigma_{i}-\sigma_{i-1}\right)
$$

is zero. Observe $\sigma_{i-1}-\sigma_{i+1}$ is nonzero by Lemma 5.6, so (61) is zero. Line (59) follows.

We obtain (60) by interchanging the roles of $\sigma_{j}, \rho_{j}(0 \leq j \leq D)$ and the roles of $\alpha, \beta$ in the above argument.

Theorem 5.8 Let $\Gamma$ denote a taut bipartite distance-regular graph with odd diameter $D \geq 5$ and valency $k \geq 3$. Let $E, F$ denote a taut pair of primitive idempotents. Let $\sigma, \rho$ denote the corresponding first cosines, respectively, and let $\alpha, \beta$ denote the corresponding weights. Then the intersection numbers of $\Gamma$ are determined by the four scalars $\sigma, \rho, \alpha, \beta$.

Proof: Given the scalars $\sigma, \rho, \alpha, \beta$, we may use (59), (60) to recursively obtain $\sigma_{i}$ and $\rho_{i}$ for $2 \leq i \leq D$. Using this information and Lemma 5.6, we set $i=1$ and $c_{1}=1$ in (11) to obtain $k$. Using (11) and Lemma 5.6, we find $c_{2}, c_{3}, \ldots, c_{D-1}$. Using $b_{i}=k-c_{i} \quad(1 \leq$ $i \leq D-1)$, we find $b_{1}, b_{2}, \ldots, b_{D-1}$.

Corollary 5.9 Let $\Gamma$ denote a taut bipartite distance-regular graph with odd diameter $D \geq 5$, valency $k \geq 3$, and eigenvalues $\theta_{0}>\theta_{1}>\cdots>\theta_{D}$. Let $d=(D-1) / 2$. Then the intersection numbers of $\Gamma$ are determined by the four scalars $k, \mu, \theta_{1}, \theta_{d}$.

Proof: Recall $E_{1}, E_{d}$ is a taut pair of primitive idempotents. Let $\sigma, \rho$ denote the corresponding first cosines, and let $\alpha, \beta$ denote the corresponding weights. By Theorem 5.8, we obtain the intersection numbers in terms of $\sigma, \rho, \alpha, \beta$. Using $\sigma=\theta_{1} / k, \rho=\theta_{d} / k$, and Corollary 5.2 (with $\theta=\theta_{1}, \theta^{\prime}=\theta_{d}$ ), we obtain $\sigma, \rho, \alpha, \beta$ in terms of $k, \mu, \theta_{1}, \theta_{d}$.

In the following lemmas, we obtain some other equations involving the cosines of a taut distance-regular graph of odd diameter.

Lemma 5.10 Let $\Gamma$ denote a taut bipartite distance-regular graph with odd diameter $D \geq$ 5 and valency $k \geq 3$. Let $E, F$ denote a taut pair of primitive idempotents. Let $\sigma_{0}, \sigma_{1}, \ldots, \sigma_{D}$ and $\rho_{0}, \rho_{1}, \ldots, \rho_{D}$ denote the corresponding cosine sequences, respectively, and let $\alpha, \beta$ denote the corresponding weights. Then

$$
\begin{gathered}
\frac{\sigma_{i-1}-\alpha \sigma_{i}}{\sigma \sigma_{i}-\sigma_{i+1}}=\frac{\beta \rho_{i}-\rho_{i+1}}{\rho \rho_{i}-\rho_{i+1}} \quad(1 \leq i \leq D-1), \\
\frac{\rho_{i-1}-\beta \rho_{i}}{\rho \rho_{i}-\rho_{i+1}}=\frac{\alpha \sigma_{i}-\sigma_{i+1}}{\sigma \sigma_{i}-\sigma_{i+1}} \quad(1 \leq i \leq D-1) .
\end{gathered}
$$

Observe the denominators in (62), (63) are nonzero by Lemma 5.6.

Proof: In the proof of Theorem 5.7, interchange the roles of $\sigma_{i-1}, \sigma_{i+1}$ and also the roles of $\rho_{i-1}, \rho_{i+1}$. 
Lemma 5.11 Let $\Gamma$ denote a taut bipartite distance-regular graph with odd diameter $D \geq$ 5 and valency $k \geq 3$. Let $E, F$ denote a taut pair of primitive idempotents. Let $\sigma_{0}, \sigma_{1}, \ldots, \sigma_{D}$ and $\rho_{0}, \rho_{1}, \ldots, \rho_{D}$ denote the corresponding cosine sequences, respectively, and let $\alpha, \beta$ denote the corresponding weights. Then

$$
\begin{aligned}
\frac{k}{b_{i}} & =\frac{\alpha \sigma_{i}-\sigma_{i-1}}{\sigma \sigma_{i}-\sigma_{i-1}}+\frac{\beta \rho_{i}-\rho_{i-1}}{\rho \rho_{i}-\rho_{i-1}} \quad(1 \leq i \leq D-1), \\
\frac{k}{c_{i}} & =\frac{\alpha \sigma_{i}-\sigma_{i+1}}{\sigma \sigma_{i}-\sigma_{i+1}}+\frac{\beta \rho_{i}-\rho_{i+1}}{\rho \rho_{i}-\rho_{i+1}} \quad(1 \leq i \leq D-1) .
\end{aligned}
$$

Observe the denominators in (64), (65) are nonzero by Lemma 5.6.

Proof: From (12), we find

$$
\begin{aligned}
\frac{k}{b_{i}} & =\frac{\sigma_{i+1}-\sigma_{i-1}}{\sigma \sigma_{i}-\sigma_{i-1}} \\
& =\frac{\alpha \sigma_{i}-\sigma_{i-1}}{\sigma \sigma_{i}-\sigma_{i-1}}+\frac{\sigma_{i+1}-\alpha \sigma_{i}}{\sigma \sigma_{i}-\sigma_{i-1}} .
\end{aligned}
$$

Evaluating the expression on the right in (67) using (59), we obtain (64). We similarly obtain (65) by using (11), (62) in place of (12), (59) in the above argument.

Definition 5.12 Let $\Gamma$ denote a bipartite distance-regular graph with diameter $D \geq 4$ and valency $k \geq 3$. We say a pair of cosine sequences of $\Gamma$ is taut whenever the associated pair of primitive idempotents is taut.

Theorem 5.13 Let $\Gamma$ denote a bipartite distance-regular graph with diameter $D \geq 4$ and valency $k \geq 3$. Let $\sigma_{0}, \sigma_{1}, \ldots, \sigma_{D}$ and $\rho_{0}, \rho_{1}, \ldots, \rho_{D}$ denote two sequences of complex scalars, and abbreviate $\sigma=\sigma_{1}, \rho=\rho_{1}$. Let $\alpha, \beta$ denote complex scalars. Then the following are equivalent:

(i) $\Gamma$ is taut and $D$ is odd. Moreover, $\sigma_{0}, \sigma_{1}, \ldots, \sigma_{D}$ and $\rho_{0}, \rho_{1}, \ldots, \rho_{D}$ form a taut pair of cosine sequences, and $\alpha, \beta$ are the weights of the corresponding primitive idempotents.

(ii) $\sigma_{0}=1, \rho_{0}=1, \sigma_{D-1}=\sigma \sigma_{D}, \rho_{D-1}=\rho \rho_{D}, \sigma \neq \alpha, \rho \neq \beta, \sigma \neq \pm \rho$, and

$$
\begin{aligned}
\frac{\sigma_{i+1}-\alpha \sigma_{i}}{\sigma \sigma_{i}-\sigma_{i-1}}=\frac{\beta \rho_{i}-\rho_{i-1}}{\rho \rho_{i}-\rho_{i-1}} \quad(1 \leq i \leq D-1), \\
\frac{\rho_{i+1}-\beta \rho_{i}}{\rho \rho_{i}-\rho_{i-1}}=\frac{\alpha \sigma_{i}-\sigma_{i-1}}{\sigma \sigma_{i}-\sigma_{i-1}} \quad(1 \leq i \leq D-1), \\
\frac{k}{b_{i}}=\frac{\alpha \sigma_{i}-\sigma_{i-1}}{\sigma \sigma_{i}-\sigma_{i-1}}+\frac{\beta \rho_{i}-\rho_{i-1}}{\rho \rho_{i}-\rho_{i-1}} \quad(1 \leq i \leq D-1),
\end{aligned}
$$

and the denominators in (68)-(70) are nonzero.

Proof: (i) $\Rightarrow$ (ii). We assume $\sigma_{0}, \sigma_{1}, \ldots, \sigma_{D}$ is a cosine sequence, so $\sigma_{0}=1$ and $\sigma_{D-1}=$ $\sigma \sigma_{D}$ by Lemma 2.1(ii). Similarly, $\rho_{0}=1$ and $\rho_{D-1}=\rho \rho_{D}$. Observe $\sigma \neq \pm \rho$ by Lemma 2.6 
and Theorem 2.17(i). Observe $\sigma \neq \alpha, \rho \neq \beta$ by Lemma 5.5. Equations (68)-(70) follow from Theorem 5.7 and Lemma 5.11.

(ii) $\Rightarrow$ (i). Let $\theta_{0}>\theta_{1}>\cdots>\theta_{D}$ denote the eigenvalues of $\Gamma$. Replacing the rightmost fraction in (70) using (68) and simplifying, we obtain

$$
b_{i}\left(\sigma_{i+1}-\sigma_{i-1}\right)=k\left(\sigma \sigma_{i}-\sigma_{i-1}\right) \quad(1 \leq i \leq D-1)
$$

We assume $\sigma_{0}=1, \sigma_{D-1}=\sigma \sigma_{D}$, so $\sigma_{0}, \sigma_{1}, \ldots, \sigma_{D}$ is a cosine sequence of $\Gamma$ by Lemma 2.1. Similarly, $\rho_{0}, \rho_{1}, \ldots, \rho_{D}$ is a cosine sequence of $\Gamma$. Let $E$ (respectively $F$ ) denote the primitive idempotent of $\Gamma$ associated with $\sigma_{0}, \sigma_{1}, \ldots, \sigma_{D}$ (respectively $\rho_{0}, \rho_{1}, \ldots, \rho_{D}$ ). Observe $E, F$ are nontrivial, since otherwise we would have a zero denominator in (68) at $i=1$ by Lemma 2.7. We show $E, F$ is a taut pair. To do this, we show (37) holds for $1 \leq i \leq D-1$. Fixing an integer $i(1 \leq i \leq D-1)$, we multiply the Eqs. (68), (69) together and simplify to obtain

$$
\left(\sigma_{i+1}-\alpha \sigma_{i}\right)\left(\rho_{i+1}-\beta \rho_{i}\right)=\left(\beta \rho_{i}-\rho_{i-1}\right)\left(\alpha \sigma_{i}-\sigma_{i-1}\right)
$$

Expanding (72), we routinely obtain (37). We have now shown (37) holds for $1 \leq i \leq D-1$. Combining this with Theorem 5.1, we find $E, F$ is a taut pair. Now the cosine sequences $\sigma_{0}, \sigma_{1}, \ldots, \sigma_{D}$ and $\rho_{0}, \rho_{1}, \ldots, \rho_{D}$ form a taut pair by Definition 5.12.

We show $\Gamma$ is taut by applying Definition 2.15 . We just showed $E, F$ is a taut pair of primitive idempotents. To show $\Gamma$ is taut, we show $\Gamma$ is not 2 -homogeneous. To do this, we assume $\Gamma$ is 2-homogeneous and obtain a contradiction. First observe $\sigma \neq 0, \rho \neq 0$; otherwise, we have a zero denominator in (68) for $i=2$. By Theorem 2.14, at least one of $E, F$ is in the set $\left\{E_{1}, E_{D-1}\right\}$. Without loss of generality, we assume $E$ is one of $E_{1}, E_{D-1}$. By Theorem 2.18, there exists a real scalar $\lambda$ such that

$$
\sigma_{i-1}-\lambda \sigma_{i}+\sigma_{i+1}=0 \quad(1 \leq i \leq D-1)
$$

Combining (73) at $i=1,2$, we find

$$
\sigma_{2}\left(\sigma_{2}+1\right)=\sigma\left(\sigma_{3}+\sigma\right)
$$

Consider the expression

$$
e=\frac{\sigma_{2}\left(\sigma_{2}+1\right)-\sigma\left(\sigma_{3}+\sigma\right)}{\sigma^{2}-\sigma_{2}}
$$

We remark that the denominator in (75) is nonzero; otherwise $\sigma^{2}-1$ is zero by Corollary 2.2 , forcing a zero denominator in (68) at $i=1$. We evaluate $e$ in two ways. First, by (74), we find $e=0$. Second, applying (68), (69), we solve for $\sigma_{2}, \sigma_{3}$ in terms of $\sigma, \rho, \alpha, \beta$. Eliminating $\sigma_{2}, \sigma_{3}$ in $e$ using this, we obtain

$$
e=\frac{(\rho-\beta)(\sigma+\rho)(\sigma-\rho)}{\rho(\rho-1)(\rho+1)} .
$$


We assumed $\rho \neq \beta, \sigma \neq \pm \rho$, so $e \neq 0$. We now have a contradiction. We conclude $\Gamma$ is not 2-homogeneous. Now $\Gamma$ is taut by Definition 2.15 .

We now show $D$ is odd. Suppose $D$ is even. By Theorem 2.17(ii), we find one of $E, F$ is $E_{d}$, where $d=D / 2$. Since the eigenvalue associated with $E_{d}$ is 0 , we find $\sigma=0$ or $\rho=0$, a contradiction. We conclude $D$ is odd. Finally, since we proved (37) holds for $1 \leq i \leq D-1$, we conclude by Corollary 5.4 that $\alpha, \beta$ are the weights for $E, F$, respectively.

In Example 2.16, we mentioned that the doubled Odd graphs are taut. Using the above theorem, we can readily verify this is the case. Our result is the following.

Example 5.14 Given an integer $k \geq 3$, let $\Gamma$ denote the graph $2 . O_{k}$ from Example 2.16. Recall $\Gamma$ is a bipartite distance-regular graph with valency $k$ and diameter $D=2 k-1$. Define a sequence of scalars $\sigma_{0}, \sigma_{1}, \ldots, \sigma_{D}$ by

$$
\sigma_{i}=\frac{(-1)^{i}+(2 k-1)(2 i-2 k+1)}{4 k(1-k)} \quad(0 \leq i \leq D) .
$$

Let $\rho_{0}, \rho_{1}, \ldots, \rho_{D}$ denote the sequence $y_{0},-y_{1}, y_{1},-y_{2}, y_{2}, \ldots,-y_{k}$, where

$$
y_{i}=(-1)^{i}\left(\begin{array}{l}
k \\
i
\end{array}\right)^{-1} \quad(0 \leq i \leq k) .
$$

Now define scalars $\alpha, \beta$ by

$$
\begin{aligned}
& \alpha=1+(k-1) k^{-2} \\
& \beta=-k^{-2} .
\end{aligned}
$$

Using (18), we may verify that the scalars $\alpha, \beta$ and the sequences $\sigma_{0}, \sigma_{1}, \ldots, \sigma_{D}$ and $\rho_{0}, \rho_{1}, \ldots, \rho_{D}$ satisfy all the conditions of Theorem 5.13(ii). Applying Theorem 5.13, we find $\Gamma$ is taut. Moreover, $\sigma_{0}, \sigma_{1}, \ldots, \sigma_{D}$ and $\rho_{0}, \rho_{1}, \ldots, \rho_{D}$ form a taut pair of cosine sequences, and $\alpha, \beta$ are the corresponding weights. We remark that the eigenvalues of $\Gamma$ associated with $\sigma_{0}, \sigma_{1}, \ldots, \sigma_{D}$ and $\rho_{0}, \rho_{1}, \ldots, \rho_{D}$ are $k-1$ and 1 , respectively.

\section{Antipodal 2-covers}

In this section we prove that any taut bipartite distance-regular graph of odd diameter $D \geq 5$ is an antipodal 2-cover. We begin with the definition of antipodal.

Definition 6.1 Let $\Gamma$ denote a distance-regular graph with vertex set $X$ and diameter $D \geq 2$. Let $\Gamma_{D}$ denote the undirected graph with vertex set $X$ and edge set $\{(x, y) \mid x, y \in$ $X, \partial(x, y)=D\}$, where $\partial$ is the path-length distance function of $\Gamma . \Gamma$ is said to be antipodal whenever $\Gamma_{D}$ is a disjoint union of cliques. In this case each clique has cardinality $1+k_{D}$, where $k_{D}$ is from (4). We refer to $\Gamma$ as an $r$-cover, where $r=1+k_{D}$. 
Lemma 6.2 [2, Prop. 4.2.2] Let $\Gamma$ denote a bipartite distance-regular graph of odd diameter $D \geq 3$. Then $\Gamma$ is antipodal if and only if $b_{i}=c_{D-i}$ for $0 \leq i \leq D$. In this case, $\Gamma$ is a 2-cover.

We mention a result about the cosine sequences of an antipodal 2-cover.

Lemma 6.3 [2, pp. 142-143] Let $\Gamma$ denote a bipartite distance-regular graph with diameter $D \geq 3$, valency $k \geq 3$, and eigenvalues $\theta_{0}>\theta_{1}>\cdots>\theta_{D}$. Assume $\Gamma$ is an antipodal 2-cover. Choose an integer $j \quad(0 \leq j \leq D)$, and let $\sigma_{0}, \sigma_{1}, \ldots, \sigma_{D}$ denote the cosine sequence of $\Gamma$ associated with $\theta_{j}$. If $j$ is even, then $\sigma_{i}=\sigma_{D-i}$ for $0 \leq i \leq D$. If $j$ is odd, then $\sigma_{i}=-\sigma_{D-i}$ for $0 \leq i \leq D$.

Theorem 6.4 Let $\Gamma$ denote a taut bipartite distance-regular graph with odd diameter $D \geq 5$ and valency $k \geq 3$. Then $\Gamma$ is an antipodal 2-cover.

Proof: Let $\theta_{0}>\theta_{1}>\cdots>\theta_{D}$ denote the eigenvalues of $\Gamma$, and let $\sigma_{0}, \sigma_{1}, \ldots, \sigma_{D}$ denote the cosine sequence associated with $\theta_{1}$. We first show

$$
\sigma_{D-j}=\sigma_{j} \sigma_{D} \quad(0 \leq j \leq D)
$$

To show (81), it is convenient to simultaneously consider the cosine sequence for $\theta_{d}$, where $d=(D-1) / 2$. Let $\rho_{0}, \rho_{1}, \ldots, \rho_{D}$ denote the cosine sequence for $\theta_{d}$. For $0 \leq j \leq D$, let $\sigma_{j}^{\prime}:=\sigma_{D-j} / \sigma_{D}$ and $\rho_{j}^{\prime}:=\rho_{D-j} / \rho_{D}$. We recall $\sigma_{D}, \rho_{D}$ are nonzero by Corollary 2.3(ii). We show $\sigma_{j}^{\prime}=\sigma_{j}, \quad \rho_{j}^{\prime}=\rho_{j}$ for $0 \leq j \leq D$. Our proof is by induction on $j$. Observe $\sigma_{0}^{\prime}=\sigma_{0}, \rho_{0}^{\prime}=\rho_{0}$ by construction and since $\sigma_{0}=\rho_{0}=1$. Observe $\sigma_{1}^{\prime}=\sigma_{1}, \quad \rho_{1}^{\prime}=\rho_{1}$ by Lemma 2.1(ii). Now fix an integer $i(1 \leq i \leq D-1)$, and assume by induction that $\sigma_{j}^{\prime}=\sigma_{j}, \rho_{j}^{\prime}=\rho_{j}$ for $0 \leq j \leq i$. We show $\sigma_{i+1}^{\prime}=\sigma_{i+1}, \rho_{i+1}^{\prime}=\rho_{i+1}$. Replacing $i$ by $D-i$ in (62), we obtain

$$
\frac{\sigma_{i+1}^{\prime}-\alpha \sigma_{i}^{\prime}}{\sigma \sigma_{i}^{\prime}-\sigma_{i-1}^{\prime}}=\frac{\beta \rho_{i}^{\prime}-\rho_{i-1}^{\prime}}{\rho \rho_{i}^{\prime}-\rho_{i-1}^{\prime}},
$$

where $\alpha, \beta$ denote the weights of $\theta_{1}, \theta_{d}$, respectively. Comparing (82), (59), and using $\sigma_{i}^{\prime}=\sigma_{i}, \sigma_{i-1}^{\prime}=\sigma_{i-1}, \rho_{i}^{\prime}=\rho_{i}, \rho_{i-1}^{\prime}=\rho_{i-1}$, we find $\sigma_{i+1}^{\prime}=\sigma_{i+1}$. Interchanging the roles of $\sigma_{0}, \sigma_{1}, \ldots, \sigma_{D}$ and $\rho_{0}, \rho_{1}, \ldots, \rho_{D}$ in the above argument, we similarly find $\rho_{i+1}^{\prime}=\rho_{i+1}$.

We have now shown $\sigma_{j}^{\prime}=\sigma_{j}$ and $\rho_{j}^{\prime}=\rho_{j}$ for $0 \leq j \leq D$. In particular, we have (81). We now show $b_{i}=c_{D-i}$ for $0 \leq i \leq D$. Assume $1 \leq i \leq D-1$; otherwise the result is trivial. Replacing $i$ by $D-i$ in (11), and evaluating the result using (81), we find

$$
c_{D-i}\left(\sigma_{i+1}-\sigma_{i-1}\right)=k\left(\sigma \sigma_{i}-\sigma_{i-1}\right)
$$

Comparing (83), (12), and using Lemma 5.6, we find $b_{i}=c_{D-i}$.

We finish this paper with a comment on Theorem 5.8. Let $\Gamma$ denote a taut bipartite distance-regular graph with odd diameter $D \geq 5$, valency $k \geq 3$, and eigenvalues $\theta_{0}>$ 
$\theta_{1}>\cdots>\theta_{D}$. Consider the taut pair $E_{1}, E_{d}$, where $d=(D-1) / 2$. Let $\sigma_{0}, \sigma_{1}, \ldots, \sigma_{D}$ and $\rho_{0}, \rho_{1}, \ldots, \rho_{D}$ denote the corresponding cosine sequences, and let $\alpha, \beta$ denote the corresponding weights. Combining Lemma 6.3 and Theorem 6.4, we find $\sigma_{D-i}=-\sigma_{i}$ and $\rho_{D-i}=(-1)^{d} \rho_{i}$ for $0 \leq i \leq D$. In particular,

$$
\sigma_{d+1}=-\sigma_{d}, \quad \rho_{d+1}=(-1)^{d} \rho_{d}
$$

In view of Theorem 5.8, the equations in (84) imply two polynomial equations involving $\sigma, \rho, \alpha, \beta$, where we abbreviate $\sigma=\sigma_{1}, \rho=\rho_{1}$. For the cases $D=5$ and $D=7$, we have obtained these equations explicitly, and they seem rather complicated. In particular, it is not clear how to use them to solve for two of $\sigma, \rho, \alpha, \beta$ in terms of the other two. We will pursue this matter in a future paper.

\section{Acknowledgments}

This paper was written while the author was a graduate student at the University of Wisconsin-Madison. The author would like to thank his advisor, Paul M. Terwilliger, for his many valuable ideas and suggestions.

\section{References}

1. E. Bannai and T. Ito, Algebraic Combinatorics I: Association Schemes, Benjamin/Cummings, London, 1984.

2. A.E. Brouwer, A.M. Cohen, and A. Neumaier, Distance-Regular Graphs, Springer-Verlag, Berlin, 1989.

3. B. Curtin, "2-homogeneous bipartite distance-regular graphs," Discrete Math. 187 (1998), 39-70.

4. G. Dickie and P. Terwilliger, "Dual bipartite Q-polynomial distance-regular graphs," Europ. J. Combin. 17 (1996), 613-623.

5. C.D. Godsil, Algebraic Combinatorics, Chapman and Hall, New York, 1993.

6. A. Jurišić and J. Koolen, "1-homogeneous graphs with Cocktail Party $\mu$-graphs," J. Alg. Combin., in press.

7. A. Jurišić and J. Koolen, "Krein parameters and antipodal tight graphs with diameter 3 and 4,” Discrete Math. 244 (2002), 181-202.

8. A. Jurišić and J. Koolen, "Nonexistence of some antipodal distance-regular graphs of diameter four," Europ. J. Combin. 21 (2000), 1039-1046.

9. A. Jurišić and J. Koolen, "A local approach to 1-homogeneous graphs," Designs, Codes, and Cryptography 21 (2000), 127-147.

10. A. Jurišić, J. Koolen, and P. Terwilliger, “Tight distance-regular graphs with small diameter," University of Ljubljana Preprint Series 36 (621), 1998.

11. A. Jurišić, J. Koolen, and P. Terwilliger, “Tight distance-regular graphs,” J. Alg. Combin. 12 (2000), $163-197$.

12. M. MacLean, “An inequality involving two eigenvalues of a bipartite distance-regular graph," Discrete Math. 225 (2000), 193-216.

13. K. Nomura, "Homogeneous graphs and regular near polygons," J. Combin. Theory Ser. B 60 (1994), 63-71.

14. K. Nomura, "Spin models on bipartite distance-regular graphs," J. Combin. Theory Ser. B 64 (1995), 300-313.

15. A. Pascasio, "An inequality in character algebras," Discrete Math., in press.

16. A. Pascasio, "An inequality on the cosines of a tight distance-regular graph," Linear Algebra Appl. 325 (2001), $147-159$.

17. A. Pascasio, "Tight distance-regular graphs and the Q-polynomial property," Graphs Combin. 17 (2001), 149-169.

18. A. Pascasio, "Tight graphs and their primitive idempotents," J. Alg. Combin. 10 (1999), 47-59.

19. M. Tomiyama, "A note on the primitive idempotents of distance-regular graphs," Discrete Math. 240 (2001), 281-294. 